\title{
Psychopathy traits in adolescents with childhood attention-deficit hyperactivity disorder
}

Tom Fowler, Kate Langley, Frances Rice, Naureen Whittinger, Kenny Ross, Stephanie van Goozen, Michael J. Owen, Michael C. O'Donovan, Marianne B. M. van den Bree and Anita Thapar

\section{Background}

Children with attention-deficit hyperactivity disorder (ADHD) are thought to be at higher risk of psychopathy. Early biological and social adversity may contribute to this risk.

\section{Aims \\ To examine psychopathy traits in ADHD.}

\section{Method}

In a sample of children with ADHD who had reached adolescence, total psychopathy and 'emotional-dysfunction' scores (e.g. callousness, lack of affect) were assessed using the Hare Psychopathy Checklist-Youth Version.

\section{Results}

A total of 156 (79\%) eligible families participated. Total psychopathy and emotional-dysfunction scores were elevated in comparison to published UK norms but none scored in the clinical range for psychopathy. Adjusting for associated conduct problems, total psychopathy scores were associated with maternal smoking during pregnancy, emotional-dysfunction scores were associated with birth complications, and neither was associated with family adversity.

\section{Conclusions}

Children with ADHD show psychopathy traits but are not 'psychopaths'. Early adversity, indexed by pre- or perinatal adversity but not family factors, appears to be associated.

\section{Declaration of interest}

Janssen-Cilag funded T.F. for 1 year.
Attention-deficit hyperactivity disorder (ADHD) is strongly associated with conduct disorder ${ }^{1,2}$ and thought also to increase risk for the development of juvenile psychopathy. ${ }^{3}$ Psychopathy traits in adolescence are important. ${ }^{4}$ They are linked to greater frequency of violent and non-violent acts of delinquency, severity of criminal acts $^{5}$ and may index meaningful heterogeneity of conduct disorder. ${ }^{6}$ The origins of psychopathy are thought to lie in childhood ${ }^{6,7}$ but relatively little is known about specific risk factors and the relationship with ADHD. We investigate psychopathy traits in a longitudinal study of children with ADHD and examine the relationship with early adversity.

\section{Methods}

\section{Participants}

Participants for this study were selected from a sample of 375 children with ADHD who had participated in a research study 5 years earlier. ${ }^{8}$ The sample had been recruited from district child and adolescent psychiatry and paediatric clinics in North England, South West England and South Wales (UK). In the UK, the diagnosis of ADHD is given by specialist (secondary care) services. Families of consecutive referrals with a suspected diagnosis of ADHD were asked by the local clinician whether they would consider participating in the study and over 95\% agreed. All the children had met diagnostic criteria for DSM-III- $\mathrm{R}^{9}$ or DSM-IV ${ }^{10}$ ADHD after a detailed research assessment, had IQ test scores of above 70 and did not have pervasive developmental disorder, Tourette syndrome or any neurological conditions.

To be eligible, participants were required to be 12 years of age or older. The eligible sample consisted of 197 adolescents (180 boys, 17 girls) aged $12-19$ years $($ mean $=14.5$ years, s.d.=1.7). Through the UK National Health Service system, 95\% of this sample was successfully traced and $79 \%(n=156)$ participated in the current study.

Ethical approval for the study was obtained from the NorthWest England Multi-Centre Research Ethics Committee, UK, and written informed consent and assent was obtained from parents and children/adolescents for the original study 5 years previously and for the current follow-up study.

\section{Measures}

The interview and questionnaire assessments used in this phase of the study were the same as those used in the original study 5 years earlier with the exception that for this study adolescent as well as parent reports of psychopathology were available and psychopathy traits were assessed.

\section{Interviews}

Parent and adolescent interviews were conducted by trained, supervised psychology graduates and a post-doctoral researcher. The parent version of the Child and Adolescent Psychiatric Assessment (CAPA), ${ }^{11}$ a semi-structured research diagnostic interview, was used to assess clinical symptoms of DSM-IV ADHD, oppositional defiant disorder and conduct disorder. The Child AttentionDeficit Hyperactivity Disorder Teacher Telephone Interview

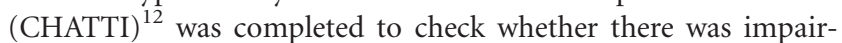
ment in more than one setting (i.e. school). The adolescent was interviewed using the child version of the CAPA that includes the same questions on antisocial behaviour, anxiety and depression but not ADHD. ${ }^{13}$ Conduct disorder and oppositional defiant disorder symptoms were considered present if reported by either parent or child. DSM-III-R/DSM-IV diagnoses were generated using information from the CAPA and the CHATTI. All interviews were audiotaped, interviewers were supervised weekly by an experienced clinician (A.T.) (for all families) and 20 audiotapes were randomly selected for re-rating and to check interrater reliability. Interrater reliability was high, $\kappa=1.00$ for DSM-IV ADHD and $\kappa=1.00$ for ICD- $10^{14}$ hyperkinetic disorder.

Psychopathy traits were assessed using the Hare Psychopathy Checklist-Youth Version ${ }^{5}$ (PCL-YV), a 20-item semi-structured interview designed and validated in a variety of settings to assess psychopathy in 12-18 year olds (each item is rated on a 3-point 
scale). It is recommended that a multisource, multi-informant approach is taken in the assessment of PCL-YV items. ${ }^{5}$ Psychopathy scores were generated using the method advised by its authors, focusing on the adolescent interview information but including a review of file information collected during the original study and the current follow-up study.

A total psychopathy score was calculated by summing all the PCL-YV items. Factor analysis of adolescent measures of psychopathy suggests that the 'emotional-dysfunction'15 component, which includes features such as callousness and a lack of remorse and affect, represents an important and distinctive factor. ${ }^{5}$ It is thought that the emotional-dysfunction component of psychopathy may have distinct biological origins as well as having a distinctive clinical presentation. ${ }^{4,7}$ Thus, emotional-dysfunction scores were additionally calculated. ${ }^{5,16}$

\section{Questionnaires}

Questionnaires were completed by mothers of participants. Information from a validated measure of pregnancy and birth complications $^{17}$ and maternal cigarette, alcohol and illicit substance use during pregnancy was collected. ${ }^{18}$ Items on this measure show very good to excellent agreement with antenatal records. ${ }^{18}$ The exceptions are length of labour and alcohol use in pregnancy, the latter item having been found to be poorly recorded in medical records. ${ }^{18}$ Family measures included family conflict and cohesion, ${ }^{19}$ the number of children in the household, social class and whether the biological parents of the child were currently separated or had ever been separated. ${ }^{20,21}$ Questionnaire information was also obtained from mothers on their current and childhood ADHD symptoms $(<18 \text { years of age })^{22}$ and from a checklist of childhood DSM-IV conduct disorder symptoms, which was summed to give a maternal childhood antisocial score.

\section{Data analysis}

Total and emotional-dysfunction psychopathy scores from the PCL-YV were transformed using natural log because of their positive skew.

Regression analysis was used to test for associations between early adversity factors and (a) total psychopathy scores and (b) emotional-dysfunction scores. We controlled for current age, use of stimulant medication at follow-up, socio-economic status and adolescent gender (where appropriate). After all analyses had been completed, multiple regression analyses were rerun using the raw outcome data (no differences were found).

The following pre- and perinatal events were examined: maternal smoking, alcohol and drug use during pregnancy, premature birth (before 37 weeks), birth weight and a composite measure of birth complications. Information on the following birth complications was available: forceps delivery, ventouse delivery, breech birth and emergency Caesarean section. Time spent in a special care baby unit or incubator was also taken as indication of birth/neonatal complications. For the purpose of analysis, individuals were divided into those who had and had not experienced birth complications, adopting the approach described by Raine et al. ${ }^{23}$ Social class was determined from the occupation of the main earner in the household (reported by parents) according to the UK Standard Occupational Classification. ${ }^{24}$ The sample was then separated into high, medium or low social class. High social class consisted of professional and managerial families $(n=41,26 \%)$, medium social class was made up of families with skilled occupations and partially skilled workers $(n=50,32 \%)$, and low social class consisted of families where the main breadwinners were in unskilled jobs or were unemployed $(n=58,37 \%)$ ( 7 families had missing data). Family factors including family conflict and cohesion scores currently and in childhood (assessed 5 years earlier), family size, relationship of biological parents (i.e. separated ever, currently separated) and social class were also examined individually and simultaneously in a forced entry multiple regression.

\section{Results}

\section{Descriptive statistics}

Overall, 156 families took part (79\%). Of these families, 9 adolescents did not wish to be interviewed. Therefore psychopathy scores were available for 147 individuals. There were no significant differences at baseline in the total number of ADHD symptoms $(t=-0.66$, d.f. $=194, \quad P=0.51)$, conduct disorder symptoms $(t=0.50$, d.f. $=195, P=0.61)$ and socio-economic status $\left(\chi^{2}=3.42\right.$, d.f. $=2, P=0.198)$ between individuals with available psychopathy scores and those without (i.e. eligible families that did not wish to take part in the follow-up as well as families where the adolescent did not wish to be interviewed). There were also no baseline clinical and socio-economic differences between families who participated and did not participate in the follow-up. ${ }^{25}$ The full clinical description of the sample (at baseline and followup) has been previously published. ${ }^{8,25}$

The original inclusion criterion for the sample was that individuals should meet either DSM-IV $(82 \%, n=128)$ or DSMIII-R (remaining 18\%, $n=28$ ) diagnostic criteria for ADHD. In this study, 5 years later, $81 \%(122 / 151)$ continued to meet full DSM-IV (54\% (82/151)) or DSM-III-R (26\% (40/151)) diagnostic criteria for ADHD and 31\% met DSM-IV diagnostic criteria for conduct disorder. ${ }^{25}$ The mean ADHD score was 14.7 at the original assessment (s.d.=2.5, range 7-18) and 12.2 at the follow-up assessment (s.d. $=4.8$, range $0-18$ ). The mean conduct disorder symptom score was 0.9 at the original assessment (s.d.=1.4, range 0-8) and 2.0 at the follow-up assessment (s.d. $=2$, range $0-8$ ).

\section{Psychopathy scores}

The mean total psychopathy score was 5.91 (s.d. $=4.21$, range $0-19$ ), significantly higher $(t=6.87, P<0.0001)$ than published mean scores (mean $=2.85$, s.d. $=2.58)$ reported in a UK community sample of 110 slightly older high school males aged $15-18$ years. ${ }^{5}$ A substantial number $(38 \%)$ scored higher than 1 standard deviation above the mean score of the UK community high school sample. This was despite the fact that over half of the adolescents were younger than 15 years, the lowest age of the high school comparison sample. Although there is no cut-point for adolescent psychopathy, ${ }^{5}$ it should be noted that none of the sample scored above cut-points previously used to categorically define psychopathy in adults. ${ }^{13}$ The mean emotional-dysfunction score was 1.00 (s.d. $=1.29$, range 0-6), higher than the mean score of 0.19 (s.d.=0.58) found in a community sample ${ }^{5}$ of adolescent males (A. Forth, personal communication, 2008).

Total psychopathy scores and emotional-dysfunction psychopathy scores were found to be significantly associated with conduct disorder symptoms $(r=0.66, P<0.01$ and $r=0.50$, $P<0.01$ respectively). Adolescents with 'high' total and emotional-dysfunction psychopathy scores $(>1$ s.d. above the mean; $n=26$ and $n=22$ respectively) were found to show higher conduct disorder symptom scores than the remainder of the sample $(t=-8.13, P<0.0001$ and $t=-6.38, P<0.0001$ respectively). Total psychopathy scores were associated with concurrent ADHD symptom severity $(\beta=0.26, t=3.16, P=0.002)$, but the association disappeared when concurrent conduct disorder was used as a covariate. No significant associations were found 
between concurrent ADHD and emotional-dysfunction scores $(\beta=0.11, t=1.35, P=0.18)$.

\section{Association of psychopathy scores with pre- and perinatal factors}

The results for pre- and perinatal factors are summarised in Table 1. Both unadjusted coefficients for each factor and adjusted coefficients (controlling for all other pre- and perinatal factors examined) are reported.

Maternal smoking $(n=60,43 \%)$ but not alcohol $(n=49,35 \%)$ or illicit drug use $(n=4,3 \%)$ during pregnancy was significantly associated with both total psychopathy scores and emotionaldysfunction scores. This association could be an artefact due to the mother's own ADHD and/or antisocial behaviour, which would increase the likelihood of their smoking during pregnancy and influence their child's behaviours. ${ }^{26,27}$ The analysis was repeated controlling for maternal conduct disorder and ADHD symptoms during childhood and maternal adult ADHD symptoms. The association of maternal smoking in pregnancy with total psychopathy scores remained significant $(\beta=0.18$, $t=2.12, \quad P=0.04$ ), but was no longer significant for the emotional-dysfunction scores $(\beta=0.15, t=1.52, P=0.13)$. Similarly when controlling for adolescent conduct disorder symptoms, the association with total psychopathy scores remained significant ( $\beta=0.18, t=2.41, P=0.02)$ but the association with emotionaldysfunction scores did not $(\beta=0.16, t=1.78, P=0.08)$.

Birth complications were associated with emotional-dysfunction scores but the association did not achieve conventional levels of significance $(P<0.05)$ for total psychopathy scores. The association with emotional-dysfunction scores remained after controlling for conduct disorder symptoms $(\beta=0.18, t=2.66$, $P=0.009$ ).

\section{Association between psychopathy scores and family adversity}

The results for family factors are summarised in Table 2. Both unadjusted coefficients for each factor and adjusted coefficients (while controlling for all other family factors) are reported. Socio-economic status, marital status of the parents and family structure were not associated with total psychopathy scores. Early family conflict (assessed 5 years earlier) but not concurrent family conflict was associated with total psychopathy scores. The association between early family conflict and total psychopathy scores dropped from significance when current ADHD severity and conduct disorder symptoms were controlled for $(\beta=0.06, t=0.77$, $P=0.44$ ). Earlier conflict also did not remain associated with psychopathy when all family factors were considered together (although this is likely to be an overly stringent test of association). Overall, the results suggest that the association seen between early family conflict and total psychopathy scores appears to be due to the relationship between psychopathy and conduct disorder symptoms. None of the family factors was associated with emotional-dysfunction scores.

\section{Discussion}

The present study is to our knowledge the first to examine juvenile psychopathy traits in a UK sample of adolescents who had had childhood ADHD.

Table 1 Associations of pre- and perinatal factors with total psychopathy scores and emotional-dysfunction scores ${ }^{a}$

\begin{tabular}{|c|c|c|c|c|c|c|c|c|c|c|c|c|}
\hline \multirow[b]{3}{*}{ Variable: pre- and perinatal factors } & \multicolumn{6}{|c|}{ Total psychopathy scores } & \multicolumn{6}{|c|}{ Emotional-dysfunction psychopathy scores } \\
\hline & \multicolumn{3}{|c|}{ Unadjusted } & \multicolumn{3}{|c|}{ Adjusted $^{b}$} & \multicolumn{3}{|c|}{ Unadjusted } & \multicolumn{3}{|c|}{ Adjusted $^{\mathrm{b}}$} \\
\hline & $\beta$ & $t$ & $P$ & $\beta$ & $t$ & $P$ & $\beta$ & $t$ & $P$ & $\beta$ & $t$ & $P$ \\
\hline Maternal smoking during pregnancy & 0.27 & 3.06 & $0.003^{* *}$ & 0.33 & 3.28 & $0.001 * *$ & 0.22 & 2.45 & $0.02 *$ & 0.27 & 2.67 & $0.009^{* *}$ \\
\hline Maternal drug use during pregnancy & -0.04 & -0.45 & 0.64 & -0.11 & -1.05 & 0.30 & -0.06 & -0.68 & 0.50 & 0.09 & 0.98 & 0.33 \\
\hline Maternal alcohol use during pregnancy & -0.02 & -0.27 & 0.79 & -0.05 & -0.53 & 0.60 & 0.07 & 0.73 & 0.47 & -0.11 & -1.09 & 0.28 \\
\hline Birth weight of child, $g$ & -0.04 & -0.48 & 0.64 & 0.06 & 0.52 & 0.61 & 0.006 & 0.07 & 0.95 & 0.07 & 0.69 & 0.50 \\
\hline Born preterm (<37 weeks) & 0.09 & 1.02 & 0.31 & 0.06 & 0.59 & 0.56 & 0.05 & 0.53 & 0.60 & 0.01 & 0.12 & 0.91 \\
\hline Birth complications & 0.21 & 2.17 & $0.03^{*}$ & 0.20 & 1.95 & 0.052 & 0.26 & 2.83 & $0.006^{* *}$ & 0.28 & 2.81 & $0.006^{* *}$ \\
\hline
\end{tabular}

\begin{tabular}{|c|c|c|c|c|c|c|c|c|c|c|c|c|}
\hline \multirow[b]{3}{*}{ Variable: family factors } & \multicolumn{6}{|c|}{ Total psychopathy scores } & \multicolumn{6}{|c|}{ Emotional-dysfunction psychopathy scores } \\
\hline & \multicolumn{3}{|c|}{ Unadjusted } & \multicolumn{3}{|c|}{ Adjusted $^{\mathrm{b}}$} & \multicolumn{3}{|c|}{ Unadjusted } & \multicolumn{3}{|c|}{ Adjusted $^{\mathrm{b}}$} \\
\hline & $\beta$ & $t$ & $P$ & $\beta$ & $t$ & $P$ & $\beta$ & $t$ & $P$ & $\beta$ & $t$ & $P$ \\
\hline Socio-economic status & 0.14 & 1.56 & 0.12 & 0.22 & 1.57 & 0.12 & 0.12 & 1.29 & 0.20 & 0.06 & 0.38 & 0.71 \\
\hline Early family conflict & 0.22 & 2.06 & $0.04^{*}$ & 0.17 & 1.04 & 0.30 & 0.12 & 1.08 & 0.28 & 0.12 & 0.66 & 0.51 \\
\hline Later family conflict & 0.10 & 1.07 & 0.29 & -0.17 & -0.91 & 0.37 & 0.007 & 0.08 & 0.94 & -0.16 & -0.81 & 0.42 \\
\hline Early family cohesion & 0.07 & 0.638 & 0.53 & -0.15 & -0.95 & 0.35 & 0.04 & 0.38 & 0.71 & -0.09 & -0.53 & 0.60 \\
\hline Later family cohesion & 0.20 & 2.02 & 0.05 & 0.13 & 0.67 & 0.51 & 0.09 & 0.92 & 0.36 & 0.16 & 0.75 & 0.46 \\
\hline Parents ever separated & 0.05 & 0.61 & 0.545 & 0.16 & 0.71 & 0.48 & 0.03 & 0.38 & 0.70 & 0.28 & 1.16 & 0.25 \\
\hline Parents currently separated & 0.14 & 1.64 & 0.10 & -0.29 & -1.19 & 0.24 & 0.04 & 0.42 & 0.67 & -0.24 & -0.95 & 0.35 \\
\hline Family size (no. of children in family) & 0.06 & 0.64 & 0.53 & 0.05 & 0.34 & 0.74 & 0.06 & 0.67 & 0.50 & -0.04 & -0.27 & 0.79 \\
\hline
\end{tabular}




\section{Psychopathy traits in ADHD}

Our sample showed elevated total psychopathy and emotionaldysfunction trait scores than a UK adolescent school sample, ${ }^{5}$ in keeping with the proposition that children with ADHD are a risk group for showing psychopathy traits. There is also some evidence from neuropsychological studies ${ }^{28}$ and a retrospective study of adult prison inmates and adults with psychopathic traits that children with $\mathrm{ADHD}^{26}$ may be 'fledgling psychopaths'. However, in our sample, the range of scores did not extend into the region in which scorers, in adulthood, are traditionally classed as 'psychopaths'.

An important consideration is that there is no established cut-off point for defining psychopathy in adolescents for the PCL-YV. ${ }^{5}$ Current adult cut-off points for psychopathy are unlikely to be appropriate for children and it is also possible that important differences in expected scores between younger and older adolescents exist. There is also evidence that total psychopathy scores vary in different countries. Compared with North American samples, there is a cultural bias towards lower scores in the UK. ${ }^{30}$ Given our findings, coupled with the uncertainty about a meaningful cut-off point for psychopathy, we suggest that it is not safe to conclude that children with ADHD grow up to be 'psychopaths'. However, children with ADHD do appear to be a group with elevated levels of total psychopathy and emotionaldysfunction traits. Rather, our findings concur with those of Piatigorsky \& Hinshaw, ${ }^{31}$ who found higher scores of psychopathy traits in a US sample of children with ADHD (aged 6-12 years) than in controls. Recent work also suggests that adolescent psychopathy is best viewed in terms of dimensional scores rather than as a category. ${ }^{32}$ Our findings also suggest that the relationship between total psychopathy scores and ADHD occurs not because of the risk effects of ADHD severity, at least within a clinical sample, but rather because of the strong links between psychopathy traits and conduct disorder symptoms.

\section{Association with early adversity}

Early biological and social adversity have been considered likely to play an important role in juvenile psychopathy ${ }^{33,34}$ but as yet there is little empirical evidence available. In the initial analyses, we found that maternal smoking during pregnancy was associated with both total psychopathy scores and the emotional-dysfunction dimension of psychopathy. There is evidence that the association of exposure to prenatal smoking with conduct disorder and antisocial behaviour may be explained by maternal characteristics, in particular maternal antisocial behaviour. ${ }^{26,27}$ In our sample the association of maternal smoking in pregnancy with total psychopathy scores was, however, not accounted for by measures of maternal antisocial and ADHD behaviours or by adolescent ADHD severity and conduct disorder symptoms. In contrast, the relationship with emotional-dysfunction psychopathy scores became non-significant when these factors were controlled for.

There is also evidence of association between maternal smoking in pregnancy and ADHD, with a pooled analysis showing a significant association with a reported odds ratio of $2.39(95 \%$ CI 1.61-3.52, $P<0.001) .{ }^{35}$ Animal studies suggest plausible mechanisms for the association ${ }^{36}$ but causality has yet to be established and the association may mainly index genetic liability. To our knowledge, there has been no previous study examining the relationship between maternal smoking in pregnancy and psychopathy traits. It is possible that previous findings of association (even if genetically mediated) between maternal smoking in pregnancy and ADHD, adult criminality and antisocial behaviour ${ }^{35,37}$ are in part driven by the relationship with psychopathy traits. This needs further investigation.
Obstetric complications have also previously been suggested as a possible contributor to developmental disturbance that, in turn, may predispose to psychopathy traits. ${ }^{34}$ Others have suggested that these may be risk factors for violent antisocial behaviour, rather than likely to be linked with the instrumental (i.e. proactive and planned) aggression characteristic of psychopathy. ${ }^{7}$ One of the few studies to examine birth complications and psychopathy found a higher incidence of obstetric complications in violent adult offenders compared with non-offender controls but not more so in those classed as psychopathic than in those classed as non-psychopathic. ${ }^{38}$ However, emotional-dysfunction scores were not specifically examined. In our study, we found an association between birth complications and emotional-dysfunction psychopathy scores, with birth complications accounting for 7\% of the variance. This finding may be important, given that there has been much interest and speculation about the role of early brain damage, especially affecting prefrontal cortical and amygdala functioning, in the aetiology of psychopathy, ${ }^{39,40}$ particularly as the lack of emotional reactivity characterised by high emotional-dysfunction psychopathy scores has been posited as the mechanism through which psychopathy may lead to elevated antisocial behaviour. ${ }^{7}$ However, there will be other pathways, including genetic liability, that can lead to altered brain function.

Finally, we found no clear evidence that psychopathy traits in ADHD are associated with family adversity. This contrasts with findings for conduct disorder, where family and social factors are established and are important correlates. Indeed in our sample, higher conduct disorder scores were associated with family adversity, specifically lower socio-economic status and higher levels of family conflict (results available from corresponding author). Moreover, there is evidence to suggest that some of these factors, notably parenting ${ }^{41}$ and poverty, ${ }^{42}$ play a causal role in childhood disruptive behaviours. The one possible exception in our sample was the observed association between family conflict in childhood (reported by mothers 5 years earlier) and total psychopathy scores (reported by adolescents). It is interesting and important that the association is found across raters and across time. However, the association appeared to be explained by associated conduct disorder symptoms.

The role of family adversity in the development of psychopathy has been uncertain. Some view the origins of psychopathy as being primarily innate, with evidence of family environment and parenting ${ }^{33,43,44}$ not being linked to antisocial behaviour in those with psychopathy or psychopathy traits. Other researchers have considered that exposure to early family adversity and disruption is likely to be important. ${ }^{45}$ Our findings suggest that it is conduct disorder symptoms rather than psychopathy traits in ADHD that are more closely associated with family adversity.

\section{Distinction of emotional-dysfunction scores}

Emotional dysfunction seen in adult, adolescent and child psychopathy has been posited to represent a distinct component of psychopathy and the mechanism through which psychopathy may lead to elevated antisocial behaviour. ${ }^{5}$ In children, the concept of 'callous-unemotional traits' has been developed to describe what is thought to be the key feature of childhood psychopathy. ${ }^{46}$ These traits bear many similarities to the emotional-dysfunction component of adult psychopathy although the concordance is not perfect. ${ }^{47,48}$ Given the potential importance of the emotional-dysfunction aspect of psychopathy as a mechanism through which psychopathy traits may develop, a key question is whether pre- and perinatal factors and family correlates for emotional-dysfunction psychopathy scores are the same as those for total psychopathy scores. The results from this 
study suggest that the pattern of correlates is similar but not identical for total and emotional-dysfunction psychopathy scores. Adjusting for associated conduct disorder symptoms, maternal smoking during pregnancy was associated with total psychopathy scores but not emotional-dysfunction scores. Birth complications were associated with emotional-dysfunction scores. These results would partially support the distinction of emotional dysfunction in terms of its link with early adversity in the form of birth complications. This increased rate of reported birth complications may of course simply index an underlying pre-existing biological and genetic vulnerability rather than represent a causal risk factor. Indeed, the evidence is that perinatal complications index underlying genetic liability for other neurodevelopmental disorders such as autism. ${ }^{49}$

\section{Limitations}

This study has several limitations which should be considered. First, the elevated psychopathy scores were not so high as to be within the range of what might be classed as 'psychopathy'. Thus, the correlates of individuals with higher psychopathy scores may differ. Although higher psychopathy scores were found than in a published sample of high school males, they were not matched geographically, by age, gender or social class. However, this was the only published community-based normative information available and the older age range of the high school sample (1518 years) makes it likely that, if anything, we have underestimated differences. Second, our findings relate to psychopathy traits in those with ADHD, and may not apply to psychopathy traits in a general or forensic population. Third, our sample size may have been insufficient to detect some small effects and given the number of potential predictive factors included in statistical tests the possibility of type 1 and type 2 errors should also be considered. The limited variation in the emotional-dysfunction scores may also have influenced results. Similarly, as we are examining a clinical sample, the restricted range in ADHD scores may attenuate the relationship with predictive factors. Fourth, it is not possible to draw conclusions as to whether the associated early factors are causal or merely indicate risk of psychopathy. Finally, despite the high rate of follow-up (79\%), differences between participants and non-participants at follow-up cannot be completely discounted, although we found no clinical and demographic differences.

\section{Conclusions}

Children with ADHD show higher than expected levels of total psychopathy traits but do not score above previously used clinical cut-points for psychopathy, and there is no association with severity of ADHD. This suggests that for community clinics, children with ADHD do not grow up to be 'psychopaths'. Preand perinatal adversity appear to be associated with total psychopathy trait scores and emotional-dysfunction scores. Family adversity seems to be linked with conduct disorder rather than psychopathy traits in ADHD.

\footnotetext{
Tom Fowler, PhD, Kate Langley, PhD, Frances Rice, PhD, Naureen whittinger Department of Psychological Medicine, Cardiff University; Kenny Ross, The FACTS Team, Young Person's Directorate, Greater Manchester West Mental Health NHS Foundation Trust, Prestwich, Manchester; Stephanie van Goozen, PhD, School of Foundation Trust, Prestwich, Manchester; Stephanie van Goozen, PhD, School of
Psychology, Cardiff University; Michael J. Owen, FRCPsych, PhD, FMedSci, Michael C. O'Donovan, FRCPsych, PhD, Marianne B. M. van den Bree, PhD, Anita Thapar, FRCPsych, PhD, Department of Psychological Medicine, Cardiff University, UK

Correspondence: Professor Anita Thapar, Department of Psychological Medicine, Cardiff University, Heath Park, Cardiff CF14 4XN, UK. Email: thapar@cf.ac.uk

First received 21 Oct 2007, final revision 5 Mar 2008, accepted 16 Jun 2008
}

\section{References}

1 Thapar A, van den Bree M, Fowler T, Langley K, Whittinger N. Predicting who develops antisocial behaviour among those with Attention Deficit Hyperactivity Disorder. Eur Child Adolesc Psychiatry 2006; 15: 118-25.

2 Mannuzza S, Klein RG, Abikoff H, Moulton JL 3rd. Significance of childhood conduct problems to later development of conduct disorder among children with ADHD: a prospective follow-up study. J Abnorm Child Psychol 2004; 32: 565-73.

3 Lynam DR. Early identification of chronic offenders: who is the fledgling psychopath? Psychol Bull 1996; 120: 209-34.

4 Pardini DA, Loeber R. Interpersonal and affective features of psychopathy in children and adolescents: advancing a developmental perspective. Introduction to special section. J Clin Child Adolesc Psychol. 2007; 36: 269-75.

5 Forth AE, Kosson DS, Hare RD. Hare Psychopathy Checklist-Youth Version: Technical Manual. Multi-Health Systems, 2003.

6 Rutter M . Commentary: What is the meaning and utility of the psychopathy concept? J Abnorm Child Psychol 2005; 33: 499-503.

7 Blair RJ, Peschardt KS, Budhani S, Mitchell DG, Pine DS. The development of psychopathy. J Child Psychol Psychiatry 2006; 47: 262-76.

8 Holmes J, Payton A, Barrett J, Harrington R, McGuffin P, Owen M, et al. Association of DRD4 in children with ADHD and comorbid conduct problems. Am J Med Genet 2002; 114: 150-3.

9 American Psychiatric Association. Diagnostic and Statistical Manual of Mental Disorders (3rd edn, revised) (DSM-III-R). APA, 1987.

10 American Psychiatric Association. Diagnostic and Statistical Manual of Mental Disorders (4th edn) (DSM-IV). APA, 1994.

11 Angold $A$, Prendergast $M$, Cox A, Harrington R, Simonoff $E$, Rutter $M$. The child and adolescent psychiatric assessment. Psychol Med 1995; 25: 739-53.

12 Holmes J, Lawson D, Langley K, Fitzpatrick H, Trumper A, Pay H, et al. The Child Attention-Deficit Hyperactivity Disorder Teacher Telephone Interview (CHATTI): reliability and validity. Br J Psychiatry 2004; 184: 74-8.

13 Angold A, Costello EJ. A test-retest reliability study of child-reported psychiatric symptoms and diagnoses using the Child and Adolescent Psychiatric Assessment (CAPA-C) Psychol Med 1995; 25: 755-62.

14 World Health Organization. Tenth Revision of the International Classification of Diseases and Related Health Problems (ICD-10). WHO, 1992.

15 Cleckley H. Mask of Sanity. Mosby, 1976.

16 Hare RD. Manual for the Hare Psychopathy Checklist - Revised (2nd edn). Multi-Health Systems, 2003.

17 Lewis SW, Murray RM. Obstetric complications, neurodevelopmental deviance, and risk of schizophrenia. J Psychiatr Res 1987; 21: 413-21.

18 Rice F, Lewis A, Harold G, van den Bree M, Boivin J, Hay DF, et al. Agreement between maternal report and antenatal records for a range of pre and perinatal factors. The influence of maternal and child characteristics. Early Hum Dev 2007; 83: 497-504.

19 Moos RH, Moos BS. Manual for the Family Environment Scale. Consulting Psychologists Press, 1981.

20 Holmes J, Payton A, Barrett JH, Hever T, Fitzpatrick H, Trumper AL, et al. A family-based and case-control association study of the dopamine D4 receptor gene and dopamine transporter gene in attention deficit hyperactivity disorder. Mol Psychiatry 2000; 5: 523-30.

21 Langley $K$, Marshall L, van den Bree M, Thomas $H$, Owen M, O'Donovan M, et al. Association of the dopamine $\mathrm{D} 4$ receptor gene 7-repeat allele with neuropsychological test performance of children with ADHD. Am J Psychiatry 2004; 161: 133-8.

22 Barkley RA. Attention Deficit Hyperactivity Disorder: A Handbook for Diagnosis and Treatment. Guilford Press, 1998.

23 Raine A, Brennan P, Mednick SA. Birth complications combined with early maternal rejection at age 1 year predispose to violent crime at age 18 years. Arch Gen Psychiatry 1994; 51: 984-8.

24 Office for National Statistics. Standard Occupational Classification (2nd edn). TSO (The Stationery Office), 1995.

25 Whittinger NS, Langley K, Fowler TA, Thomas HV, Thapar A. Clinical precursors of adolescent conduct disorder in children with attention-deficit/ hyperactivity disorder. J Am Acad Child Adolesc Psychiatry 2007; 46: 179-87.

26 Maughan B, Taylor A, Caspi A, Moffitt TE. Prenatal smoking and early childhood conduct problems: testing genetic and environmental explanations of the association. Arch Gen Psychiatry 2004; 61: 836-43.

27 Silberg JL, Parr T, Neale MC, Rutter M, Angold A, Eaves L. Maternal smoking during pregnancy and risk to boys' conduct disturbance: an examination of the causal hypothesis. Biol Psychiatry 2003; 15: 130-5. 
28 Lynam DR Early identification of the fledgling psychopath: locating the psychopathic child in the current nomenclature. J Abnorm Psychol 1998; 107: $566-75$.

29 Johansson P, Kerr M, Andershed H. Linking adult psychopathy with childhood hyperactivity-impulsivity-attention problems and conduct problems through retrospective self-reports. J Personal Disord 2005; 19: 94-101.

30 Cooke DJ, Michie C, Hart SD, Clark D. Assessing psychopathy in the UK: concerns about cross-cultural generalisability. Br J Psychiatry 2005; 186: 335-41.

31 Piatigorsky A, Hinshaw SP. Psychopathic traits in boys with and without attention-deficit/hyperactivity disorder: concurrent and longitudinal correlates. J Abnorm Child Psychol 2004; 32: 535-50.

32 Murrie DC, Marcus DK, Douglas KS, Lee Z, Salekin RT, Vincent G. Youth with psychopathy features are not a discrete class: a taxometric analysis. I Child Psychol Psychiatry 2007; 48: 714-23.

33 Viding E. Understanding the development of psychopathy. J Child Psychol Psychiatry 2004; 45: 1329-37.

34 Lykken DT. The Antisocial Personalities. Lawrence Erlbaum Associates, 1995

35 Langley $\mathrm{K}$, Rice F, van den Bree MB, Thapar A. Maternal smoking during pregnancy as an environmental risk factor for attention deficit hyperactivity disorder behaviour. A review. Minerva Pediatr 2005; 57: 359-71.

36 Ernst M, Moolchan ET, Robinson ML. Behavioural and neural consequences of prenatal exposure to nicotine. J Am Acad Child Adolesc Psychiatry 2001 40: $630-41$.

37 Brennan PA, Grekin ER, Mednick SA. Maternal smoking during pregnancy and adult male criminal outcomes. Arch Gen Psychiatry 1999; 56: 215-9.

38 Lalumiere ML, Harris GT, Rice ME. Psychopathy and developmental instability. Evol Hum Behav 2001; 22: 75-92.
39 Viding E, Williamson DE, Hariri AR. Developmental imaging genetics: challenges and promises for translational research. Dev Psychopathol 2006; 18: $877-92$.

40 Blair RJ. The amygdala and ventromedial prefrontal cortex in morality and psychopathy. Trends Cogn Sci 2007; 11: 387-92.

41 Patterson GR, DeGarmo DS, Knutson N. Hyperactive and antisocial behaviours: comorbid or two points in the same process? Dev Psychopathol 2000; 12: 91-106.

42 Costello EJ, Compton SN, Keeler G, Angold A. Relationships between poverty and psychopathology: a natural experiment. JAMA 2003; 290: 2023-9.

43 Devita E, Forth A, Hare RD. Family background of male criminal psychopaths Canadian Psychology 1990; 31: 346

44 Wooton JM, Frick PJ, Shelton KK, Silverthorn P. Ineffective parenting and childhood conduct problems. The moderating role of callous-unemotional traits. J Consult Clin Psychol 1997; 65: 301-8.

45 Farrington DP. The importance of child and adolescent psychopathy. $J$ Abnorm Child Psychol 2005; 33: 489-97.

46 Frick PJ, O'Brien BS, Wootton JM, McBurnett K. Psychopathy and conduct problems in children. J Abnorm Psychol 1994; 103: 700-7.

47 Lee Z, Vincent GM, Hart SD, Corrado RR. The validity of the Antisocial Process Screening Device as a self-report measure of psychopathy in adolescent offenders. Behav Sci Law 2004; 21: 771-86.

48 Murrie DC, Cornell DG. Psychopathy screening of incarcerated juveniles: a comparison of measures. Psychol Assess 2002; 14: 390-6.

49 Bolton PF, Murphy M, Macdonald H, Whitlock B, Pickles A, Rutter M Obstetric complications in autism: consequences or causes of the condition? J Am Acad Child Adolesc Psychiatry 1997; 36: 272-81.

\section{Psychiatrists in 19th-century fiction}

\section{The System of Dr Tarr and Professor Fether (1845), Edgar Allan Poe}

\author{
Fiona Subotsky
}

Edgar Allan Poe (1809-1849) was a popular American writer who specialised in poems and tales of gloom and horror. In England he was considered to be an example of degeneracy by Henry Maudsley in 1860, who, while appreciating Poe's melancholy genius, remarked about him: 'of all men who have walked upon the earth, it is scarcely possible to point to one whose history discloses more of folly and more of wretchedness ....'

In the story 'The System of Dr Tarr and Professor Fether' there is a classic 'gothic' description of a first approach to the threatening castle, mansion or, in this case, lunatic asylum:

'Through this dank and gloomy wood we rode ... when the Maison de Santé came in view. It was a fantastic château, much dilapidated, and indeed scarcely tenantable through age and neglect. Its aspect inspired me with absolute dread ... '

Curiously, this tone is very similar to that of the reforming medico-psychologist John Connolly, Maudsley's father-in-law, who remarked of private asylums in 1856 that they were 'generally distinguishable from all the houses in the neighbourhood by their dismal appearance: their exterior was as gloomy as their interior was dirty'.

However, once inside, there is a change of mood. The narrator is made welcome and is entertained at a bizarrely jolly banquet, where the tunes are strangely discordant and the guests decoratively but somewhat oddly dressed. Several blackened figures then burst in. It turns out that, taking advantage of the new 'soothing' system the lunatics had taken over the asylum, under the leadership of the superintendent who had become insane; the keepers were locked up ... after having been tarred and feathered. The scene reminds one of the asylum balls which were recorded and depicted at the time, and while the effect is on the whole humorous, there are reminders of the macabre similar to Poe's 'The Fall of the House of Usher' and 'The Dance of the Red Death'.

The narrator remarks that despite searching he could find no trace of the works of Professor Fether or Dr Tarr 